Tạp Chí Tin Học và Diểu Khiển Học

Tâp X(1994), Số 2, 43-48

(1)

\title{
Tính lồi - lõm của hàm sản xuất một mức với độ co giãn thay thế không đổi
}

Tô Cẩm Tú

Viẹn Quy Hoặch \& Thiết Kể Nông Nghiệp

Bọ Nông Nghiệp

\section{Mở đầu}

Ta đã biết $[1,2]$ điểu kiện cân và đủ để một hàm uhiếu biến

$$
y=f\left(x_{1}, x_{2}, \ldots x_{n}\right) \quad \text { an }
$$

lổi (lổi trên) trong một miền nào đỏ là dạng toàn phương tương ứng

$$
d^{2} y=\sum_{i=1}^{n} \sum_{j=1}^{n} \frac{\partial^{2} y}{\partial x_{i} \partial x_{j}} d x_{i} d x_{j}
$$

xác định không dương trong miển đó. Cái đó tương đương với vấn để là mọi định thức con cấp $k(k=\overline{1, n})$ của ma trận Hertz các đạo hàm riêng hạng hai

$$
H^{(n)}=\left\|\begin{array}{cccc}
\frac{\partial^{2} y}{\partial x_{1}^{2}} & \frac{\partial^{2} y}{\partial x_{1} \partial x_{2}} & \cdots & \frac{\partial^{2} y}{\partial x_{1} \partial x_{n}} \\
\frac{\partial^{2} y}{\partial x_{2} \partial x_{1}} & \frac{\partial^{2} y}{\partial x_{2}^{2}} & \cdots & \frac{\partial^{2} y}{\partial x_{2} \partial x_{n}} \\
\vdots & \vdots & \ddots & \vdots \\
\frac{\partial^{2} y}{\partial x_{n} \partial x_{1}} & \frac{\partial^{2} y}{\partial x_{2} \partial x_{n}} & \cdots & \frac{\partial^{2} y}{\partial x_{n} \partial x_{n}}
\end{array}\right\|
$$

lẩn lượt đồi dấu khi $k$ lẩn lượt nhận các giả trị từ 1 đến $n$, trong đó $\frac{\partial^{2} y}{\partial x_{1}^{2}} \leq 0$. Nói cách khác định thức

$$
(-1)^{k}\left|H^{(k)}\right| \geq 0 \text { với } k=\overline{1, n}
$$


Tuy nhiên công cụ này khá cồng kềnh, nên người ta đã có nhiêu phưnựthộkhác

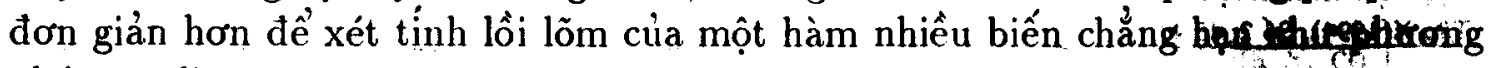
pháp gradient...

Riêng đối với hàm sản xuất $y=f\left(x_{1}, x_{2}, \ldots x_{n}\right)$, trong đó $x_{j}(j=\overline{1, n})$ là cạ́c yếu tố sản xuất và $y$ là kết quả sàn xuất, gần đây không yêu câu nó phải lổi trở cẩ miên kinh tế $\left(x_{j} \geq x_{j}^{(0)} \geq 0, j=\overline{1, n}\right)$, mà có thề lồi trong một miển con nào đó và lõm trong miển con khác của nền kinh tế $[3,4]$.

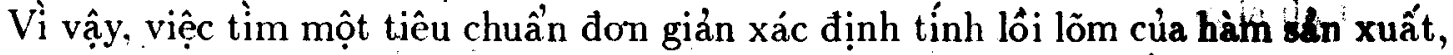
đặc biệt của hàm sản xuất với độ co dã̃n thay thế không đồi (vì do kự'đợn giàn của chúng các hàm sản xuất dạng này đã được sử dụng rất rộng rãi để thó phỏng nền kinh tế) trờ nên rất cần thiết.

Trong [4] đã chứng minh định lý cơ bản sau Cần và đủ đề hàqm sản xuất (1) có độ co dân thay thế không đồi $\sigma_{i j}=\sigma, i, j=\overline{1, n}$ là

$$
\begin{aligned}
& \delta_{k i}=\delta_{k j}
\end{aligned}
$$

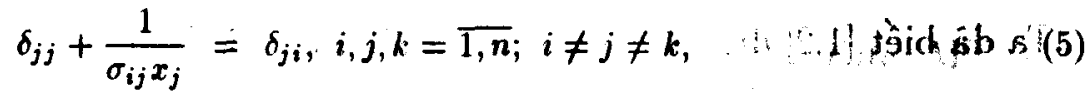

trong đó $\sigma_{i j}$ là độ co dãn thay thế $x_{i}$ và $x_{j}$

$$
\delta_{j j}=\frac{\partial^{2} y}{\partial x_{j}^{2}} / \frac{\partial y}{\partial x_{j}} \quad \ldots \text { angit iồl) }
$$

là nhịp độ tăng đơn năng xuất giới hạn;

$$
\delta_{j i}=\frac{\partial^{2} y}{\partial x_{j} \partial x_{i}} / \frac{\partial y}{\partial x_{i}}
$$

là nhịp độ tăng hốn hợp năng xuất tới hạn theo sự tăng cúa cảc yếu tố san xuất (không loại trừ thời gian).

Trên cơ sở các biều thức (4) và (5) ta có định lý sau

Dịnh lý. Hàm sản xuất với độ co dân thay thế không đổi $\sigma$

$$
y=f\left(x_{1}, x_{2}, \ldots x_{n}\right)
$$

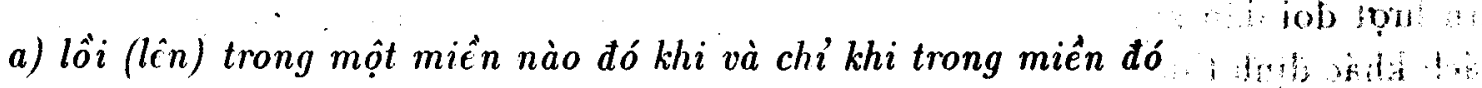

$$
\sum_{t=1}^{k} \delta_{t 2} x_{t} \leq-\frac{k-1}{\sigma}
$$




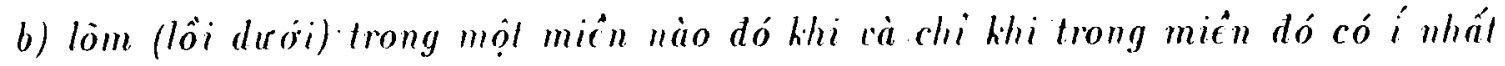
mọt

$$
\frac{\partial^{2} y}{\partial x_{j}^{2}}=0, j \in\{1,2, \ldots, n\}
$$

$v \dot{a}$

$$
\sum_{t=1}^{k} \delta_{t 1} x_{t} \geq-\frac{k-1}{\sigma}
$$

nớ $\operatorname{mọik}=\overline{1, n} ;\{1,2, \ldots, k\} \subset\{1,2, \ldots, n\}$.

Chưng minh. Tir (5), (6) và (7) ta có

$$
\begin{gathered}
\frac{\partial^{2} y}{\partial r_{j}^{2}}=\delta_{j j} \frac{\partial y}{\partial x_{j}} \\
\frac{\partial^{2} y}{\partial x_{j} \partial x_{i}}=\delta_{j i} \frac{\partial y}{\partial x_{i}}=\left(\delta_{j j}+\frac{1}{x_{j} \sigma}\right) \frac{\partial y y}{\partial x_{i}} .
\end{gathered}
$$

Nhờ đó ma trận Hertz (3) trờ thành

$$
\cdot H^{(n)}=\left\|\begin{array}{cccc}
\delta_{11} \frac{\partial y}{\partial r_{1}} & \left(\delta_{11}+\frac{1}{r_{1} \sigma}\right) \frac{\partial y}{\partial x_{2}} & \ldots & \left(\delta_{11}+\frac{1}{x_{1} \sigma}\right) \frac{\partial y}{\partial x_{n}} \| \\
\left(\delta_{22}+\frac{1}{r_{2} \sigma}\right) \frac{\partial y}{\partial x_{1}} & \delta_{22} \frac{\partial \omega_{1}}{\partial x_{2}} & \ldots & \left(\delta_{22}+\frac{1}{x_{2} \sigma}\right) \frac{\partial y}{\partial x_{n}} \\
\vdots & \vdots & \ddots & \vdots \\
\left(\delta_{n n}+\frac{1}{x_{n} \sigma}\right) \frac{\partial y}{\partial x_{1}} & \left(\delta_{n n}+\frac{1}{r_{n} \sigma}\right) \frac{\partial y}{\partial r_{2}} & \cdots & \delta_{n n} \frac{\partial y}{\partial r_{n}}
\end{array}\right\| .
$$

Tư đó dể dàng thấy rằng định thi̛ con cáp $k(1 \leq k \leq n)$ bằng

$$
\left|H^{(k)}\right|-\left(\prod_{t=1}^{k} \frac{\partial y}{\partial x_{t}}\right) \mid \begin{array}{cccc}
\delta_{11} & \delta_{1 !}+\frac{1}{x_{1} \sigma} & \ldots & \delta_{11}+\frac{1}{x_{1} \sigma} \\
\delta_{22}+\frac{1}{r_{2} \sigma} & \delta_{22} & \ldots & \delta_{22}+\frac{1}{x_{2} \sigma} \\
\delta_{k k}+\frac{1}{x_{k} \sigma} & \delta_{k k}+\frac{1}{x_{k} \sigma} & \ldots & \vdots \\
\delta_{k,} \cdot 1
\end{array}
$$

Hay

$$
\left(\prod_{t=1}^{k} \frac{\sigma x_{t} / \partial y}{\partial x_{t}}\right)\left|H^{(k)}\right|= \begin{cases}\sigma \sum_{t=1}^{k} \delta_{t t} x_{t}+(k-1), & \text { nếu } k \text { lè } \\ -\left[\sigma \sum_{t=1}^{k} \delta_{t} x_{t}+(k-1)\right]: & \text { nếu } k \text { chằn. }\end{cases}
$$

Từ tiên clề $\frac{\partial y}{\partial x_{t}} \geq 0, t=T, n$ và vì $0 \leq \sigma<\infty$ suỵ ra rằng

$$
\prod_{t=1}^{k} \sigma x_{t} / \frac{\partial y}{\partial x_{t}} \geq 0
$$

với mọi t. Vì vậy đề cho (4) thóa màn thì tư (\$) phải có

$$
\sum_{t=1}^{k} \delta_{t} x_{t} \leq-\frac{k-1}{\sigma}
$$


đương nhiên là từ (8) suy ra điểu kiện cân và đủ để

$$
(-1)^{k}\left|H^{(k)}\right| \mid \leq 0, \text { với } k=\overline{1, n}
$$

là

$$
\sum_{t=1}^{k} \delta_{t t} x_{t} \geq-\frac{k-1}{\sigma}
$$

Đề tiền đề $\partial^{2} y / \partial x_{j}^{2} \leq 0,(j=\overline{1, n})$ của hàm sản xuất cũng thỏa mãn, chỉ cân thêm một điểu kiện $\delta_{11}=0$. Nhưng vai trò các biến $x_{1}, x_{2}, \ldots, x_{n}$ là như nhau về vị trí trong biêu thức $y=f\left(x_{1}, x_{2}, \ldots x_{n}\right)$, nên chì cân ît nhất một $\delta_{j j}=0$, hay $\partial^{2} y / \partial x_{j}^{2}=0, j \in\{1,2, \ldots n\}$. Dịnh lý đã được chứng minh.

\section{3. Ứng dụng}

Ớ đây chúng ta xét một số hàm sản xuất với độ co dãn thay thế không đởi. Trước hết xét ba hàm mà nhịp độ hỗn hợp tăng năng suất tới hạn bằng không (xem [4])

1) Hàm $y=\sum_{j=1}^{n} \alpha_{j} \frac{\sigma}{\sigma-1} x_{j}^{\frac{\sigma-1}{\sigma}}$ lồi ngặt trong toàn miền $x_{j} \geq 0, j=\overline{1, n}$. Thực vậy, $\delta_{11}=-1 /\left(\sigma x_{1}\right)$, từ đó $\delta_{11} x_{1}=-1 / \sigma<0$,

$$
\delta_{11} x_{1}+\delta_{22} x_{2}+\ldots+\delta_{k k} x_{k}=-k / \sigma<-(k-1) / \sigma
$$

với mọi $k=\overline{1, n}$.

2) Hàm $y=\sum_{j=1}^{n} \alpha_{j} \ln x_{j}$ lồi ngặt vì

$$
\delta_{11} x_{1}+\delta_{22} x_{2}+\ldots+\delta_{k k} x_{k}=-k<-(k-1)
$$

3) Hàm $y=\sum_{j=1}^{n} \alpha_{j} x_{j}(\sigma=\infty)$ lồi (đồng thời lòm) vì

$$
\delta_{11} x_{1}+\delta_{22} x_{2}+\ldots+\delta_{k k} x_{k}=0
$$

với mọi $k=\overline{1, n}$.

Bây giờ ta xét một vài dạng khác cuia hàm sàn xuất với độ co dãn thay thể không đồi $[4,5]$ và NDTNSTH hồn hợp khác không.

1 - Xét hàm

$$
y=c \prod_{j=1}^{n} a_{j}^{\alpha_{i} \frac{\alpha}{\sigma-1} x_{j}^{\frac{c-1}{\sigma}}} .
$$

ta có

$$
\delta_{i j}=\alpha_{j} x_{j}^{-\frac{1}{\sigma}} \ln a_{j}-\frac{1}{\pi r_{i}} .
$$


Từ đó suy ra rằng hàm sàn xuất đà cho lồi trong miền $x_{j} \geq 0, j=\overline{1, n}$ thóa màn diều kiện

$$
y=\prod_{j=1}^{n} a_{j}^{\alpha_{i} x_{j}^{\frac{0-1}{\sigma}}} \leq e^{\frac{1}{\sigma}} .
$$

2 - Hàm Cobb - Douglass lồi nếu $0 \leq \alpha_{j} \leq 1$ và $\sum_{j=1}^{n} \alpha_{j} \leq 1$. Thực vậy, tị̛

$$
y=\prod_{j=1}^{n} \cdot r_{j}^{\alpha_{j}}
$$

ta được $\delta_{11} x_{1}=\alpha_{1}-1<0$, nếu $0<\alpha_{1}<1$. Tiếp tục,

$$
\delta_{11} x_{1}+\delta_{23} x_{2}+\ldots+\delta_{k k} x_{k}=\sum_{t=1}^{k} \alpha_{t}-k \leq-(k-1)
$$

vơi $k=\overline{1 . n}$, nốu $\sum_{\alpha_{1}} \leq 1$

3 - Hàm logistic

$$
y=\frac{a}{1+\cos p\left(-\sum_{j=1}^{n} \alpha_{j} \frac{\sigma}{\pi-1} x_{j}^{\frac{\sigma-1}{\sigma}}\right)}
$$

cho ta

$$
\delta_{j j} x_{j}=\frac{a_{j} x_{j}^{\frac{\sigma-1}{\sigma}}\left[\operatorname{ces} p\left(-\sum_{t=1}^{k} \alpha_{t} \frac{\sigma}{\sigma-1} x_{t}^{\frac{\sigma-1}{\sigma}}\right)-1\right]}{1+\operatorname{cesp}\left(-\sum_{t=1}^{n} \alpha_{t} \frac{\sigma}{\sigma-1} x_{t}^{\frac{\sigma-1}{\sigma}}\right)}-\frac{1}{\sigma} .
$$

Dê dàng thấy rằng $\delta_{j j} x_{j}<0$ khi $\sum_{t=1}^{n} a_{t} \frac{g}{\sigma-1} x_{t}^{\frac{\sigma-1}{\tau}}>\ln c$ không yêu câu chặt chẽ về giới hạn đưới, có thề thấy rằng $\delta_{j j} x_{j}<0$ khi $x_{j} \geq\left(\frac{\sigma-1}{\sigma x_{j}} \ln c\right)^{\frac{\sigma}{\sigma-1}}$ với mọi $j \in\{1,2, \ldots, n\}$.

\section{Tài liệu tham kháo}

1. James M. Henderson \& Richard E. Quand. Microc conomic Theory, A Mathematical Aproach. Second Edition 1971, Appendix 430 p.

2. Ivanov U. P. \& Lotov A. B., Mathematical models in tconomics, M., Nauka, 1979, 303 p. (in Russian).

3. Tỉnh hỉnh kinh tế ở các nước tư bản và các nước đang phát triề. MEIMO, 1987 (phần tiếp theo) (in Russian).

1. Tô Câm Tú. l'êt mộl vài phương phóp xây dựng hàm sản xuất, Trung tâm tính toán VHLKH Liên Xô, 1988, 28 p. (in Russian).

5. To Cam Tu, Concavity and converity of one - level production functions, The $4^{\text {th }}$ Congress of Vietnamese Mathematicians, Ilanoi September 4- 7, 1990, Abstract, p. 133 .

\section{Abstract}


Concavity and convexity of one - level production functions with constant elasticity of substitution

On the base of the theorem [4]: Necessary and sufficient conditions for the constancy of elasticity of substitution $\left(\sigma_{i j}=\sigma\right)$ of one - level production functions $y=f\left(x_{1}, x_{2}, \ldots, x_{n}\right)$ is $\delta_{j j}+1 /\left(\sigma_{i j} x_{j}\right)=\delta_{j i}$, where $\delta_{i j}=\frac{\partial^{2} y}{\partial x_{j} \partial x_{i}} / \frac{\partial y}{\partial x_{i}}, i, j=\overline{1, n}$ the following theorem is followed:

In the region $\Omega=\left\{x_{j} \geq 0, j=\overline{1, n}\right\}$ the one - level production functions with constant clasticity of substitution $\sigma$ is:

a. Convex in $\Omega_{1} \subset \Omega$ if and only if $\sum_{t=1}^{k} \delta_{t t} x_{t} \leq(1-k) / \sigma$.

b. Concave in $\Omega_{1}$ if and only if in this region to say the least of it $\frac{\partial^{2} y}{\partial x_{j}^{2}}=0, j \in$ $\{1,2, \ldots, n\}$, and $\sum_{t=1}^{k} \delta_{t 1} x_{t} \geq(1-k) / \sigma$, where $\{1,2, \ldots, k\} \subset\{1,2, \ldots, n\}$ and $k=\frac{j}{1, n}$. 